\title{
Spin Symmetry for Anti-Lambda Spectrum in atomic nucleus*
}

\author{
SONG Chun-Yan, ${ }^{1}$ YAO Jiang-Ming, ${ }^{1,2}$ and MENG Jie ${ }^{\dagger 1,3,4,5}$ \\ ${ }^{1}$ School of Phyics, and State Key Laboratory of Nuclear \\ Physics and Technology, Peking University, Beijing, 100871 \\ ${ }^{2}$ School of Physics, Southwest University, Chongqing, 400715 \\ ${ }^{3}$ Center of Theoretical Nuclear Physics, \\ National Laboratory of Heavy Ion Accelerator, Lanzhou 730000 \\ ${ }^{4}$ Institute of Theoretical Physics, Chinese Academy of Sciences, Beijing 100080 \\ ${ }^{5}$ Department of Physics, University of Stellenbosch, Stellenbosch, South Africa
}

(Dated: October 30, 2018)

\begin{abstract}
The spin symmetry of anti-Lambda spectrum in nucleus ${ }^{16} \mathrm{O}$ has been studied in the relativistic mean field theory. The spin-orbit splittings of spin doublets are found to be around 0.03-0.07 MeV and the dominant components of the Dirac spinor for the anti-Lambda spin doublets are found to be near identical. It indicates that there is an even better spin symmetry in the anti-Lambda spectrum than that in the anti-nucleon spectrum.
\end{abstract}

PACS numbers: 21.80.+a, 21.10.Hw, 21.30.Fe, 21.10.Pc

\footnotetext{
* Supported by the National Key Basic Research Programme of China under Grant No 2007CB815000, the National Natural Science Foundation of China under Grant Nos 10775004, the Southwest University Initial Research Foundation Grant to Doctor (No. SWU109011). Helpful discussions with Avraham Gal are gratefully acknowledged.

$\dagger$ Email: mengj@pku.edu.cn
} 
Symmetries in single particle spectrum of atomic nuclei have been discussed extensively in the literature, as the violation of spin-symmetry by the spin-orbit term and approximate pseudo-spin symmetry in nuclear single particle spectrum: atomic nuclei are characterized by a very large spin-orbit splitting, i.e., pairs of single particle states with opposite spin $\left(j=l \pm \frac{1}{2}\right)$ have very different energies[1]. This fact has allowed the understanding of magic numbers in nuclei and forms the basis of nuclear shell structure. More than thirty years ago pseudo-spin quantum numbers have been introduced by $\tilde{l}=l \pm 1$ and $\tilde{j}=j$ for $j=l \pm \frac{1}{2}$ and it has been observed that the splitting between pseudo-spin doublets in nuclear single particle spectrum is by an order of magnitude smaller than the normal spin-orbit splitting [2, 3].

The relativistic mean field (RMF) theory has been widely used for describing nuclear matter, finite nuclei and hypernuclei [4]. Since the relation between the pseudospin symmetry and the RMF theory was first noted in Ref.[5], the RMF theory has been extensively used to describe the pseudospin symmetry in the nucleon spectrum. In Refs. [6], it suggested that the origin of pseudospin symmetry is related to the strength of the scalar and vector potentials. Ginocchio took a step further to reveal that pseudo-orbital angular momentum is nothing but the "orbital angular momentum" of the lower component of the Dirac wave function, and showed clearly that the origin of pseudo-spin symmetry in nuclei is given by a relativistic symmetry in the Dirac Hamiltonian[7]. The quality of pseudo-spin symmetry has been found to be related to the competition between the centrifugal barrier and the pseudo-spin orbital potential [8, 9] with the RMF theory.

The possibility of producing a new nuclear system with one or more anti-baryons inside normal nuclei has recently gained renewed interest [10, 11, 12, 13, 14]. In Ref.[15], the RMF theory has been used to investigate the antinucleon spectrum, which corresponds to the negative energy solutions to the Dirac equation, and a well developed spin symmetry has been found in the antinucleon spectrum. It motivates the present study of the spin symmetry in the single $\bar{\Lambda}$ spectrum, which can provide us important information on the antiparticles and their interaction with nuclei.

In the RMF theory, the $\bar{\Lambda}$ hyperon is described as a Dirac spinor moving in the potentials generated by the meson fields,

$$
\left\{\boldsymbol{\alpha} \cdot \boldsymbol{p}+V_{\bar{\Lambda}}(\boldsymbol{r})+\beta\left[M_{\bar{\Lambda}}+S_{\bar{\Lambda}}(\boldsymbol{r})\right]\right\} \psi_{\bar{\Lambda}}(\boldsymbol{r})=\epsilon_{\bar{\Lambda}} \psi_{\bar{\Lambda}}(\boldsymbol{r}),
$$

where $M_{\bar{\Lambda}}$ is the mass of $\bar{\Lambda}$ and chosen as $M_{\bar{\Lambda}}=1115.7 \mathrm{MeV}, \epsilon_{\bar{\Lambda}}$ is the single-particle energy. 
As $\bar{\Lambda}$ is a charge neutral and isoscalar particle, it couples only to the $\sigma$ and $\omega$ mesons. As a consequence, the scalar $S_{\bar{\Lambda}}(\boldsymbol{r})$ and vector $V_{\bar{\Lambda}}(\boldsymbol{r})$ potentials are given by,

$$
\begin{aligned}
S_{\bar{\Lambda}}(\boldsymbol{r}) & =g_{\sigma \bar{\Lambda}} \sigma(\boldsymbol{r}), \\
V_{\bar{\Lambda}}(\boldsymbol{r}) & =g_{\omega \bar{\Lambda}} \omega(\boldsymbol{r}) .
\end{aligned}
$$

According to the charge conjugation transformation, the coupling constants for $\bar{\Lambda}$ are related to those for $\Lambda$ by the following relations,

$$
\begin{aligned}
& g_{\sigma \bar{\Lambda}}=g_{\sigma \Lambda} \\
& g_{\omega \bar{\Lambda}}=-g_{\omega \Lambda} .
\end{aligned}
$$

For a spherical system, the Dirac spinor of $\bar{\Lambda}$ is characterized with quantum numbers $\{n l \kappa m\}$ and has the form

$$
\psi_{\bar{\Lambda}}(\boldsymbol{r})=\frac{1}{r}\left(\begin{array}{c}
i G_{n \kappa}(r) Y_{j m}^{l}(\theta, \phi) \\
-F_{\tilde{n} \kappa}(r) Y_{j m}^{\tilde{l}}(\theta, \phi)
\end{array}\right), \quad j=l \pm \frac{1}{2},
$$

where $Y_{j m}^{l}(\theta, \phi)$ are the spin spherical harmonics, $G_{n \kappa}(r) / r$ and $F_{\tilde{n} \kappa}(r) / r$ form the radial wave functions for the upper and lower components with $n$ and $\tilde{n}$ radial nodes, and $\kappa=$ $\langle 1+\boldsymbol{\sigma} \cdot \mathbf{l}\rangle=(-1)^{j+l+1 / 2}(j+1 / 2)$ characterizes the spin orbit operator and the quantum numbers $l$ and $j$.

Using the relation between the upper and lower components, one can obtain the Schrödinger-like equations for the upper (dominant) component from Eq.(1),

$$
\left[-\frac{1}{2 M_{+}}\left(\frac{d^{2}}{d r^{2}}+\frac{1}{2 M_{+}} \frac{d V_{-}}{d r} \frac{d}{d r}-\frac{l(l+1)}{r^{2}}\right)-\frac{1}{4 M_{+}^{2}} \frac{\kappa}{r} \frac{d V_{-}}{d r}+M_{\bar{\Lambda}}-V_{+}\right] G(r)=\epsilon_{\bar{\Lambda}} G(r),
$$

where $M_{+}=M_{\bar{\Lambda}}+\epsilon_{\bar{\Lambda}}-V_{-}$and $V_{ \pm}(r)=V_{\bar{\Lambda}}(r) \pm S_{\bar{\Lambda}}(r)$. In Eq. (7), it is shown that the spin symmetry depends on the competition between the spin-orbit term (the term $\sim \kappa$ ) and the centrifugal term [8]. In the following, the spin symmetry in $\bar{\Lambda}$ spectrum in atomic nucleus will be studied by taking ${ }^{16} \mathrm{O}$ as an example.

With the mean-field and no-sea approximations, the coupled Dirac equations for nucleons and $\bar{\Lambda}$ together with the Klein-Gordon equations for mesons can be self-consistently solved by iteration. The effective interaction PK1 [16] is adopted for the nucleon part, and the 


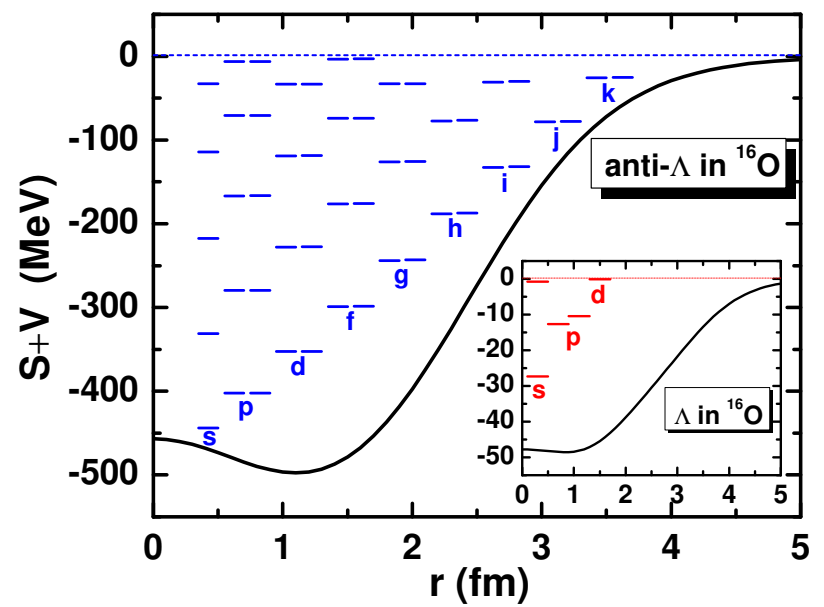

FIG. 1: Potential and spectrum of $\bar{\Lambda}$ in ${ }^{16} \mathrm{O}$. For each pair of the spin doublets, the left levels are with $\kappa<0$ and the right ones with $\kappa>0$. The inset gives the potential and spectrum of $\Lambda$ in ${ }^{16} \mathrm{O}$.

coupling constants for $\bar{\Lambda}$ are chosen as, $g_{\sigma \bar{\Lambda}}=g_{\sigma \Lambda}=\frac{2}{3} g_{\sigma N}$ and $g_{\omega \bar{\Lambda}}=-g_{\omega \Lambda}=-\frac{2}{3} g_{\omega N}$, according to the $\mathrm{SU}(3)$ symmetry in naive quark model.

The potential and single $\bar{\Lambda}$ spectrum in ${ }^{16} \mathrm{O}$ are plotted in Fig. 1, where for each pair of the spin doublets, the left levels are with $\kappa<0$ and the right ones with $\kappa>0$. For comparison, the potential and single $\Lambda$ spectrum in ${ }^{16} \mathrm{O}$ are given as well. As seen in Fig. 1, the single anti-Lambda energies for each spin doublets are almost identical, and the energy differences between spin doublets $\epsilon_{\bar{\Lambda}\left(n l_{j=l-1 / 2}\right)}-\epsilon_{\bar{\Lambda}\left(n l_{j=l+1 / 2}\right)}$ in the anti-Lambda spectrum are around $0.09-0.17 \mathrm{MeV}$ for p states, which are much smaller than that in Lambda spectrum, $2.26 \mathrm{MeV}$.

In order to see the splitting size and its energy dependence more clearly, the $\bar{\Lambda}$ spin-orbit splitting,

$$
\Delta E_{s . o}=\left(\epsilon_{\bar{\Lambda}\left(n l_{j=l-1 / 2}\right)}-\epsilon_{\bar{\Lambda}\left(n l_{j=l+1 / 2}\right)}\right) /(2 l+1),
$$

in ${ }^{16} \mathrm{O}$ is plotted as a function of the the average energy,

$$
E_{\mathrm{av}}=\frac{1}{2}\left(E_{\bar{\Lambda}\left(n l_{j=l-1 / 2}\right)}+E_{\bar{\Lambda}\left(n l_{j=l+1 / 2}\right)}\right)
$$

in Fig. 2, where $E_{\bar{\Lambda}}=\epsilon_{\bar{\Lambda}}-M_{\bar{\Lambda}}$. For comparison, the spin-orbit splittings for antineutron are also plotted here. It is seen that the spin-orbit splittings $\Delta E_{\text {s.o }}$ for p states in antiLambda spectrum are around 0.03-0.06 MeV, which is much smaller than those both in Lambda spectrum, $0.75 \mathrm{MeV}$, and in antineutron spectrum, 0.09-0.18 MeV. It indicates that an even better spin symmetry in $\bar{\Lambda}$ spectrum has been found than that in antineutron 


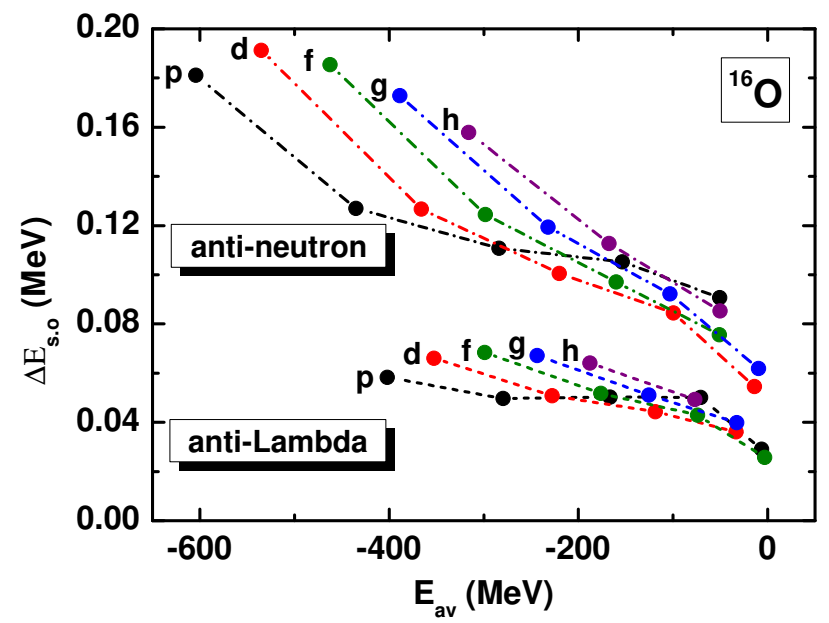

FIG. 2: The spin-orbit splitting $\Delta E_{\text {s.o }}$ [defined in Eq.(8)] for $\bar{\Lambda}$ and antineutron in ${ }^{16} \mathrm{O}$ as a function of the average energy $E_{\mathrm{av}}$ [defined in Eq.(9)]. For each orbit $l$, the radial quantum numbers $n$ of the spin doublets are $1,2, \cdots$, respectively from left to right.

spectrum [15]. This can be easily understood from $V_{-}^{\bar{\Lambda}}(r)=\frac{2}{3} V_{-}^{\bar{n}}(r)$, and $M_{+}^{\bar{\Lambda}}>M_{+}^{\bar{n}}$ due to $M_{+}^{\bar{\Lambda}}=M_{\bar{\Lambda}}-\left(-\epsilon_{\bar{\Lambda}}+V_{-}^{\bar{\Lambda}}\right), M_{+}^{\bar{n}}=M_{\bar{n}}-\left(-\epsilon_{\bar{n}}+V_{-}^{\bar{n}}\right)$, and $M_{\bar{\Lambda}}>M_{\bar{n}},-\epsilon_{\bar{\Lambda}}<-\epsilon_{\bar{n}}$, where $M_{\bar{n}}$ is the mass of anti-neutron. Therefore the spin-orbit term $\frac{1}{4 M_{+}^{2}} \frac{\kappa}{r} \frac{d V_{-}}{d r}$ in the Eq. (7) for $\bar{\Lambda}$ is smaller than two thirds of that for antineutron, which leads to an even better spin symmetry in $\bar{\Lambda}$ spectrum than that in antineutron ones. Moreover, it is found that the spin-orbit splittings for other orbits are around 0.03-0.07 MeV, which decreases as the levels approaching to the continuum.

In Fig. 3, we plot the radial wave functions $G(r)$ and $F(r)$ for several $\bar{\Lambda}$ spin doublets in ${ }^{16} \mathrm{O}$ with different orbit quantum numbers. Since the spin-orbit splitting in the single $\bar{\Lambda}$ spectrum in ${ }^{16} \mathrm{O}$ is so small, the dominant component $G(r)$ of the wave functions of the spin doublets are nearly identical while the small components $F(r)$ are quite different. The relation between the node numbers of large and small components for the spin-doublets [15, 17] can be clearly seen in Fig. 3, i.e.,

$$
\tilde{n}=n+1, \text { for } \kappa>0 ; \tilde{n}=n, \text { for } \kappa<0 \text {. }
$$

In summary, taking ${ }^{16} \mathrm{O}$ as an example, the spin symmetry in the single $\bar{\Lambda}$ spectrum has been studied within the RMF theory. The spin-orbit splittings in the $\bar{\Lambda}$ spectrum have been found to be around 0.03-0.07 MeV, which are much smaller than those in antineutron spectrum 0.06-0.20 MeV. The reasons are due to the shallower potential and larger mass 


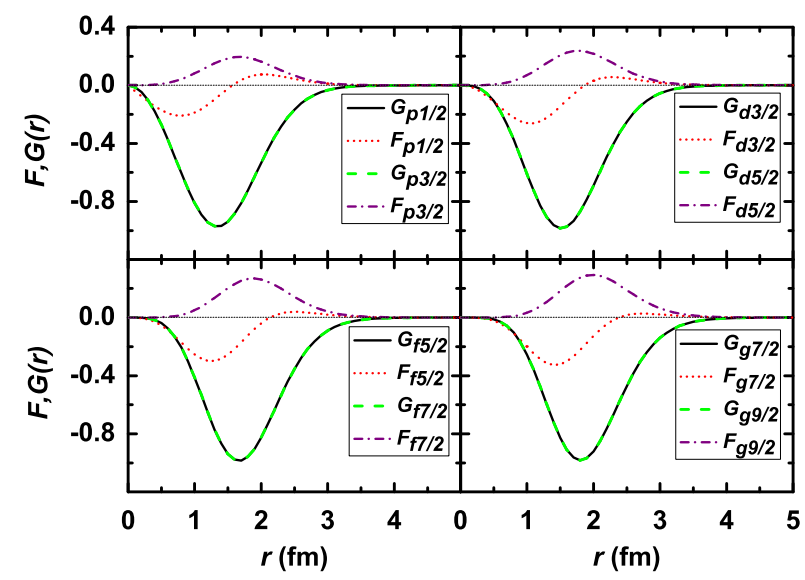

FIG. 3: Radial wave functions of $\bar{\Lambda}$ spin doublets with different orbit quantum numbers in ${ }^{16} \mathrm{O}$.

for $\bar{\Lambda}$ hyperon. The dominant components of the Dirac spinor for the $\bar{\Lambda}$ spin doublets are found to be near identical. All these show that an even better spin symmetry than that in the anti-nucleon spectrum [15] can be expected in the anti-Lambda spectrum.

It has to be pointed out that, as in the anti-nucleon case [15], the imaginary part of the optical potential of anti-Lambda has not been taken into account here, which is large and may make it difficult to observe the spin-orbit splitting of the anti-Lambda levels experimentally. In addition, the polarization effects due to the $\bar{\Lambda}$ is neglected here. For a real $\bar{\Lambda}-{ }^{16} \mathrm{O}\left(\overline{\bar{\Lambda}}^{17} \mathrm{O}\right)$ system, the mean fields including the scalar and vector ones will be modified by the added $\bar{\Lambda}[10]$. The study of spin symmetry in anti-Lambda spectrum with these self-consistences is in progress.

It is known that the tensor coupling will cancel with the original spin-orbit potential and give a small the spin-orbit splitting in Lambda hypernucleus [18, 19, 20]. For anti-Lambda hypernuclei, although the tensor coupling is expected to increase the spin-orbit splitting, the conclusion that spin symmetry in anti-Lambda is better than that in anti-neutron will remain true as the contribution of the tensor couplings is comparable in magnitude with the original spin-orbit potential for $\bar{\Lambda}$. The detailed quantitative studies of this effect will be given elsewhere.

[1] Mayer M G and Jensen J H D 1955 Elementary Theory of Nuclear Shell Struture (New York: Wiley) 
[2] Arima A, Harvey M, and Shimizu K 1969 Phys. Lett. 30B 517

[3] Hecht K and Adler A 1969 Nucl. Phys. A137 129

[4] Meng J et al 2006 Prog. Part. Nucl. Phys. 57470

[5] Bahri C, Draayer J P, and Moszkowski S A 1992 Phys. Rev. Lett. 682133

[6] Blokhin A L, Bahri C, and Draayer J P 1995 Phys. Rev. Lett. 744149

[7] Ginocchio J N 1997 Phys. Rev. Lett. 78 436; 2005 Phys. Rep. 414 165; and references therein

[8] Meng J et al 1998 Phys. Rev. C58 R628

[9] Meng J et al 1999 Phys. Rev. C59 154

[10] Bürvenich T et al 2002 Phys. Lett. B542 261

[11] Mishustin I N et al 2005 Phys. Rev. C71 035201

[12] Friedman E, Gal A, and Mareš J 2005 Nucl. Phys. A761 283

[13] Larionov A B et al 2008 Phys. Rev. C78 014604

[14] Larionov A B et al 2009 arXiv:nucl-th/0903.2152

[15] Zhou S G, Meng J, and Ring P 2003 Phys. Rev. Lett. 91262501

[16] Long W H et al 2004 Phys. Rev. C69 034319.

[17] Leviatan A and Ginocchio J N 2001 Phys. Lett. B518 214

[18] Jennings B K 1990 Phys. Lett. B246 325

[19] Noble J V 1980 Phys. Lett. B89 325

[20] Yao J M et al 2008 Chin. Phys. Lett. 251629 